\title{
Algebraical criteria of stability for delayed memristive neural networks
}

\author{
Ailong $\mathrm{Wu}^{1,2,3^{*}}$ and Zhigang Zeng ${ }^{3}$
}

${ }^{\text {*Correspondence: }}$

hbnuwu@yeah.net

${ }^{1}$ College of Mathematics and

Statistics, Hubei Normal University,

Huangshi, 435002, China

${ }^{2}$ Institute for Information and

System Science, Xi'an Jiaotong

University, Xi'an, 710049, China

Full list of author information is

available at the end of the article

\begin{abstract}
Memristive neurodynamic systems find many potential applications in mixed analog-digital multichip neurogrid and integrated photo-supercapacitor nanotube arrays. Analysis and design of memristive neurodynamic systems have attracted a large amount of research interest. In this study, some new neurodynamic approaches are proposed for stability analysis of delayed memristive neural networks. Some less conservative stability criteria are established by considering the memristor multiport effect, which is ignored in the previous literature. Numerical examples are given to demonstrate the effectiveness of these stability criteria.
\end{abstract}

Keywords: memristive neurodynamic systems; hybrid systems; switched network cluster; stability

\section{Introduction}

In recent years, memristive neurodynamic systems have become one of the most widely researched topics in the computing architecture enabled by memristors [1-16]. A multiport memristive neurodynamic system is a promising electronic system for its neuromorphic memristor memory [5]. It is worth mention that using memristors as synaptic connections in neuromorphic electronic systems has been suggested with different neural architectures. One of the main characteristics hindering the neural architecture of memristive neuromorphic systems is the sneak-paths phenomenon. Consequently, various interesting nonlinear properties of memristive neuromorphic systems have emerged [1-4, 6-16].

Recently, some works $[1,3,6-9,13-15]$ have demonstrated the interest in studying global stability for some types of memristive neurodynamic systems, such as fractionalorder memristive neurodynamic systems [1] and integral-order memristive neurodynamic systems $[3,6-9,13-15]$. Periodic oscillation in neurodynamic systems is an interesting dynamic effect, furthermore, the analysis of persistent oscillation for memristive neurodynamic systems is able to reveal crucial features of the dynamics $[2,16]$. In an associative memory model based on memristors, multiple stable states are necessary. The multistability of memristive neurodynamic systems has been investigated in $[4,10]$. To control the memristive physiological states, some control strategies of exponential stabilization for memristive neurodynamic systems are proposed in [11]. Consider, for example, the dynamic-state sequence objects where, under the universal need of multi-attractor, the multi-object flow closely relates to a multi-attractor of the network cluster. Thus, Wu and Zeng [12] discussed the Lagrange stability of memristive neurodynamic systems with various feedback functions.

(c) 2015 Wu and Zeng; licensee Springer. This is an Open Access article distributed under the terms of the Creative Commons Attribution License (http://creativecommons.org/licenses/by/4.0), which permits unrestricted use, distribution, and reproduction in any medium, provided the original work is properly credited. 
Despite some clear progress on the qualitative analysis of memristive neurodynamic systems, the issue of the memristor multiport effect has not been fully used in the existing studies, except $[1,2,13]$, when dealing with the qualitative analysis of such systems. As is well known, the memristor multiport effect explains how intelligent behavior arises from neural models. Ignoring the memristor multiport effect in developing computing platforms based on memristive neuromorphic systems is very unreasonable. Once abandoning the memristor multiport effect, designers of these systems face a major problem: whether such neuromorphic system can accurately simulate memristive intelligent behavior in a mixed analog-digital integrated circuit.

In this paper, we consider the global exponential stability and global asymptotical stability for a class of delayed memristive neural networks. The analytical method differs from those considered in most of the existing literature on qualitative analysis of memristive neurodynamic systems, where the memristor multiport effect is ignored. The obtained algebraical criteria, which can be directly derived from the network parameters, are easily testable. Consequently, a physical realization of such a system is rather simple.

The rest of this paper is organized as follows. Section 2 presents the network model and preliminaries. Section 3 details the analytical framework and theoretical results. Section 4 provides two illustrative examples. Section 5 gives the concluding remarks.

\section{Model description and preliminaries}

Based on $[1,13]$, in this paper, consider a class of delayed memristive neural networks described by the following delay differential equations: for $i=1,2, \ldots, n$,

$$
\dot{x}_{i}(t)=-d_{i} x_{i}(t)+\sum_{j=1}^{n} a_{i j}\left(x_{i}(t)\right) f_{j}\left(x_{j}\left(t-\tau_{i j}\right)\right)+u_{i},
$$

where $x_{i}(t)$ denotes the memristive neuron state, $d_{i}>0$ is the self-inhibition, $0 \leq \tau_{i j} \leq \tau$ ( $\tau \geq 0$ is a constant) is the transmission delay, feedback function $f_{i}(\cdot)$ is bounded and $f_{i}(0)=0, u_{i}$ denotes the external input, $a_{i j}\left(x_{i}(t)\right)$ is the connection memristive weight, which is defined as

$$
a_{i j}\left(x_{i}(t)\right)= \begin{cases}\hat{a}_{i j}, & x_{i}(t)>0 \\ \check{a}_{i j}, & x_{i}(t)<0\end{cases}
$$

for $i, j=1,2, \ldots, n$, where $\hat{a}_{i j}$ and $\check{a}_{i j}$ are constants.

The initial condition of neurodynamic system (1) is assumed to be

$$
x(t)=\left(x_{1}(t), x_{2}(t), \ldots, x_{n}(t)\right)^{T}=\phi(t)=\left(\phi_{1}(t), \phi_{2}(t), \ldots, \phi_{n}(t)\right)^{T}, \quad t_{0}-\tau \leq t \leq t_{0},
$$

where $\phi_{i}(t) \in \mathcal{C}\left(\left[t_{0}-\tau, t_{0}\right], \Re\right), i=1,2, \ldots, n$.

Let $x_{t} \in \mathcal{C}\left(\left[t_{0}-\tau, t_{0}\right], \Re^{n}\right)$ be defined by $x_{t}(s)=x(t+s), t_{0}-\tau \leq s \leq t_{0}$, and (3) can be rewritten as

$$
x_{t_{0}}=\phi \in \mathcal{C}\left(\left[t_{0}-\tau, t_{0}\right], \Re^{n}\right) .
$$


In addition, we also assume that the feedback function $f_{i}(\cdot)(i=1,2, \ldots, n)$ satisfies the Lipschitz condition with the Lipschitz constant $l_{i}>0$, i.e.,

$$
\left|f_{i}(\chi)-f_{i}(\widehat{\chi})\right| \leq l_{i}|\chi-\widehat{\chi}|, \quad i=1,2, \ldots, n, \forall \chi, \widehat{\chi} \in \Re
$$

Remark 1 From a systems-theoretic point of view, neurodynamic system (1) is basically a state-dependent switched network cluster. For analyzing and controlling the statedependent switched network cluster, nonsmooth analysis will be devoted to dealing with neurodynamic system (1). The theory and application of conventional nonlinear systems have been extensively studied over the past few decades; see [17-40]. However, little attention has been paid to the switched network cluster [41].

In this paper, solutions of all the systems considered are in the following interpreted in Filippov's sense. $K(\mathcal{P})$ denotes closure of the convex hull of set $\mathcal{P} \cdot \operatorname{co}\{\widetilde{\Pi}, \widehat{\Pi}\}$ denotes closure of the convex hull generated by real numbers $\widetilde{\Pi}$ and $\widehat{\Pi}$. Let $\bar{a}_{i j}=\max \left\{\hat{a}_{i j}, \check{a}_{i j}\right\}, \underline{a}_{i j}=$ $\min \left\{\hat{a}_{i j}, \check{a}_{i j}\right\}, \tilde{a}_{i j}=\max \left\{\left|\hat{a}_{i j}\right|,\left|\check{a}_{i j}\right|\right\}$, for $i, j=1,2, \ldots, n$.

When considering neurodynamic system (1), throughout this paper, let us define the set-valued maps as follows:

$$
K\left(a_{i j}\left(x_{i}(t)\right)\right)= \begin{cases}\hat{a}_{i j}, & x_{i}(t)>0, \\ \operatorname{co}\left\{\hat{a}_{i j}, \check{a}_{i j}\right\}, & x_{i}(t)=0, \\ \check{a}_{i j}, & x_{i}(t)<0 .\end{cases}
$$

Obviously, for $i, j=1,2, \ldots, n$,

$$
\operatorname{co}\left\{\hat{a}_{i j}, \check{a}_{i j}\right\}=\left[\underline{a}_{i j}, \bar{a}_{i j}\right]
$$

By the theory of differential inclusions, from (1), for $i=1,2, \ldots, n$,

$$
\dot{x}_{i}(t) \in-d_{i} x_{i}(t)+\sum_{j=1}^{n} K\left(a_{i j}\left(x_{i}(t)\right)\right) f_{j}\left(x_{j}\left(t-\tau_{i j}\right)\right)+u_{i}
$$

A solution $x(t)=\left(x_{1}(t), x_{2}(t), \ldots, x_{n}(t)\right)^{T}$ in the sense of Filippov of neurodynamic system (1), with initial condition $x(s)=\phi(s), s \in\left[t_{0}-\tau, t_{0}\right]$, is absolutely continuous on any compact interval of $\left[t_{0},+\infty\right)$, and

$$
\dot{x}_{i}(t) \in-d_{i} x_{i}(t)+\sum_{j=1}^{n} K\left(a_{i j}\left(x_{i}(t)\right)\right) f_{j}\left(x_{j}\left(t-\tau_{i j}\right)\right)+u_{i}
$$

Remark 2 Filippov solutions are mainly used to analyze nonlinear switched systems. On the concept of Filippov solutions, one may refer to the well-known publication [41].

Definition 1 A constant vector $x^{*}=\left(x_{1}^{*}, x_{2}^{*}, \ldots, x_{n}^{*}\right)^{T}$ is called an equilibrium point of neurodynamic system (1), if for $i=1,2, \ldots, n$,

$$
0 \in-d_{i} x_{i}^{*}+\sum_{j=1}^{n} K\left(a_{i j}\left(x_{i}^{*}\right)\right) f_{j}\left(x_{j}^{*}\right)+u_{i}
$$


Definition 2 The equilibrium point $x^{*}=\left(x_{1}^{*}, x_{2}^{*}, \ldots, x_{n}^{*}\right)^{T}$ of neurodynamic system (1) is said to be globally exponentially stable, if there exist constants $\kappa>0$ and $\tilde{\kappa}>0$ such that

$$
\left|x_{i}\left(t, t_{0}, x_{0}\right)-x_{i}^{*}\right| \leq \kappa\left|x_{i}(0)-x_{i}^{*}\right| \exp \left\{-\tilde{\kappa}\left(t-t_{0}\right)\right\}, \quad \forall t \geq t_{0}, i=1,2, \ldots, n .
$$

Definition 3 The equilibrium point $x^{*}=\left(x_{1}^{*}, x_{2}^{*}, \ldots, x_{n}^{*}\right)^{T}$ of neurodynamic system (1) is said to be globally asymptotically stable, if it is locally stable and globally attractive.

The following two lemmas play an important role in discussing the qualitative behavior of neurodynamic system (1).

Lemma 1 For neurodynamic system (1) there exists at least one equilibrium point.

In subsequent sections, we will denote by $x^{*}=\left(x_{1}^{*}, x_{2}^{*}, \ldots, x_{n}^{*}\right)^{T}$ the equilibrium point of (1).

Lemma 2 For neurodynamic system (1),

$$
\left|K\left(a_{i j}\left(x_{i}\right)\right) f_{j}\left(x_{j}\right)-K\left(a_{i j}\left(y_{i}\right)\right) f_{j}\left(y_{j}\right)\right| \leq \tilde{a}_{i j} l_{j}\left|x_{j}-y_{j}\right|, \quad \forall i, j=1,2, \ldots, n, \forall x_{i}, y_{i} \in \Re .
$$

Lemmas 1 and 2 can be proved using standard arguments as the Lemmas 1 and 2 in [13], respectively.

Remark 3 Lemma 2 of this paper makes good use of the memristor multiport effect. It establishes a kind of internal ties between system attribute and its neurodynamic approaches.

\section{Main results}

According to Lemma 1, neurodynamic system (1) has the equilibrium point $x^{*}=\left(x_{1}^{*}, x_{2}^{*}, \ldots\right.$, $\left.x_{n}^{*}\right)^{T}$; we shift the equilibrium point $x^{*}=\left(x_{1}^{*}, x_{2}^{*}, \ldots, x_{n}^{*}\right)^{T}$ to the origin by the translation $z(t)=x(t)-x^{*}$ in the differential inclusion (6), which results in

$$
\dot{z}_{i}(t) \in-d_{i} z_{i}(t)+\sum_{j=1}^{n} K\left(a_{i j}\left(z_{i}(t)\right)\right) f_{j}\left(z_{j}\left(t-\tau_{i j}\right)\right), \quad i=1,2, \ldots, n
$$

where

$$
K\left(a_{i j}\left(z_{i}(t)\right)\right) f_{j}\left(z_{j}\left(t-\tau_{i j}\right)\right)=K\left(a_{i j}\left(z_{i}(t)+x_{i}^{*}\right)\right) f_{j}\left(z_{j}\left(t-\tau_{i j}\right)+x_{j}^{*}\right)-K\left(a_{i j}\left(x_{i}^{*}\right)\right) f_{j}\left(x_{j}^{*}\right) .
$$

According to Lemma 2,

$$
\left|K\left(a_{i j}\left(z_{i}(t)\right)\right) f_{j}\left(z_{j}\left(t-\tau_{i j}\right)\right)\right| \leq \tilde{a}_{i j} l_{j}\left|z_{j}\left(t-\tau_{i j}\right)\right|
$$

From (7)-(9),

$$
\left|\dot{z}_{i}(t)\right| \leq-d_{i}\left|z_{i}(t)\right|+\sum_{j=1}^{n} \tilde{a}_{i j} l_{j}\left|z_{j}\left(t-\tau_{i j}\right)\right|, \quad i \in\{1,2, \ldots, n\} .
$$


Theorem 1 The equilibrium point $x^{*}=\left(x_{1}^{*}, x_{2}^{*}, \ldots, x_{n}^{*}\right)^{T}$ of neurodynamic system (1) is globally exponentially stable, if any one of the conditions (11) and (12) holds:

$$
\begin{aligned}
& d_{i}>\sum_{j=1}^{n} \tilde{a}_{i j} l_{j}, \quad i=1,2, \ldots, n, \\
& d_{i}>\sum_{j=1}^{n} \tilde{a}_{j i} l_{i}, \quad i=1,2, \ldots, n .
\end{aligned}
$$

Proof Consider the comparison systems of (10)

$$
\left|\dot{\tilde{z}}_{i}(t)\right|=-d_{i}\left|\tilde{z}_{i}(t)\right|+\sum_{j=1}^{n} \tilde{a}_{i j} l_{j}\left|\tilde{z}_{j}\left(t-\tau_{i j}\right)\right|, \quad i \in\{1,2, \ldots, n\},
$$

where $\tilde{z}_{i}\left(t_{0}, z_{0}\right)=z_{i}\left(t_{0}\right), i=1,2, \ldots, n$.

According to the comparison theorem [22],

$$
\left|z_{i}\left(t, t_{0}, z_{0}\right)\right| \leq\left|\tilde{z}_{i}\left(t, t_{0}, z_{0}\right)\right|, \quad \forall t \geq t_{0}, i=1,2, \ldots, n .
$$

When (11) holds, choose a sufficiently small positive constant $\rho>0$ such that

$$
d_{i}-\rho>\sum_{j=1}^{n} \tilde{a}_{i j} l_{j}, \quad i=1,2, \ldots, n .
$$

Let $\hat{z}_{i}(t)=\exp \{\rho t\} \tilde{z}_{i}(t), i=1,2, \ldots, n$. Then

$$
\left|\dot{\hat{z}}_{i}(t)\right|=\left(-d_{i}+\rho\right)\left|\hat{z}_{i}(t)\right|+\sum_{j=1}^{n} \tilde{a}_{i j} l_{j}\left|\hat{z}_{j}\left(t-\tau_{i j}\right)\right|, \quad i \in\{1,2, \ldots, n\},
$$

where $\hat{z}_{i}\left(t_{0}\right)=\exp \left\{\rho t_{0}\right\} z_{i}\left(t_{0}\right), i=1,2, \ldots, n$.

Based on the work in [17], it follows that the zero solution of (15) is globally asymptotically stable if and only if the conditions (16) and (17) hold:

$$
\begin{aligned}
& {\left[\left(-d_{i}+\rho\right) \delta_{i j}+\tilde{a}_{i j} l_{j}\right]_{n \times n} \text { is a Hurwitz matrix, }} \\
& \operatorname{det}\left[\left(i \omega \delta_{i j}+\left(d_{i}-\rho\right)\right) \delta_{i j}-\tilde{a}_{i j} l_{j} \exp \left\{-i \omega \tau_{i j}\right\}\right]_{n \times n} \neq 0, \quad \forall \omega \in \Re,
\end{aligned}
$$

where

$$
\delta_{i j}= \begin{cases}1, & i=j \\ 0, & i \neq j, i, j=1,2, \ldots, n .\end{cases}
$$

Applying the Groshgorin circular disc theorem [22], by (14), it implies that (16) is true. On the other hand,

$$
\begin{aligned}
\left|i \omega+d_{i}-\rho\right|-\sum_{j=1}^{n} \tilde{a}_{i j} l_{j}\left|\exp \left\{-i \omega \tau_{i j}\right\}\right| & \geq\left|\operatorname{Re}\left(i \omega+d_{i}-\rho\right)\right|-\sum_{j=1}^{n} \tilde{a}_{i j} l_{j}\left|\exp \left\{-i \omega \tau_{i j}\right\}\right| \\
& =\left|d_{i}-\rho\right|-\sum_{j=1}^{n} \tilde{a}_{i j} l_{j}>0, \quad i=1,2, \ldots, n .
\end{aligned}
$$


Combining with (14) and the Groshgorin circular disc theorem [22], (17) holds, then the zero solution of (15) is globally asymptotically stable. Meanwhile,

$$
\begin{aligned}
& \left|z_{i}\left(t, t_{0}, z_{0}\right)\right| \leq\left|\tilde{z}_{i}\left(t, t_{0}, z_{0}\right)\right|=\exp \left\{-\rho\left(t-t_{0}\right)\right\}\left|\hat{z}_{i}\left(t, t_{0}, \exp \left\{\rho t_{0}\right\} z_{0}\right)\right|, \\
& \quad \forall t \geq t_{0}, i=1,2, \ldots, n .
\end{aligned}
$$

Therefore, the zero solution of (7) is globally exponentially stable, i.e., the equilibrium point $x^{*}=\left(x_{1}^{*}, x_{2}^{*}, \ldots, x_{n}^{*}\right)^{T}$ of $(1)$ is globally exponentially stable.

When (12) holds, the proof can be presented using standard arguments similar to the proof above.

Theorem 2 The equilibrium point $x^{*}=\left(x_{1}^{*}, x_{2}^{*}, \ldots, x_{n}^{*}\right)^{T}$ of neurodynamic system (1) is globally asymptotically stable, if any one of the conditions (18) and (19) holds:

$$
\begin{aligned}
& d_{i} \geq \sum_{j=1}^{n} \tilde{a}_{j i} l_{i}, \quad i=1,2, \ldots, n, \\
& d_{i} \geq \sum_{j=1}^{n} \tilde{a}_{i j} l_{j}, \quad i=1,2, \ldots, n .
\end{aligned}
$$

Proof When (18) holds, consider the following functional:

$$
V(t)=\sum_{i=1}^{n}\left|z_{i}(t)\right|+\sum_{i=1}^{n} \sum_{j=1}^{n} \int_{t-\tau_{i j}}^{t} \tilde{a}_{i j} l_{j}\left|z_{j}(\vartheta)\right| \mathrm{d} \vartheta .
$$

Calculating the upper right Dini derivative of $V(t)$ along the solution $z\left(t, t_{0}, z_{0}\right)$ of (7),

$$
\begin{aligned}
D^{+} V(t) \leq & -\sum_{i=1}^{n} d_{i}\left|z_{i}(t)\right|+\sum_{i=1}^{n} \sum_{j=1}^{n} \tilde{a}_{i j} l_{j}\left|z_{j}\left(t-\tau_{i j}\right)\right|+\sum_{i=1}^{n} \sum_{j=1}^{n} \tilde{a}_{i j} l_{j}\left|z_{j}(t)\right| \\
& -\sum_{i=1}^{n} \sum_{j=1}^{n} \tilde{a}_{i j} l_{j}\left|z_{j}\left(t-\tau_{i j}\right)\right| \\
= & -\sum_{i=1}^{n} d_{i}\left|z_{i}(t)\right|+\sum_{i=1}^{n} \sum_{j=1}^{n} \tilde{a}_{i j} l_{j}\left|z_{j}(t)\right| \\
= & \sum_{i=1}^{n}\left[-d_{i}+\sum_{j=1}^{n} \tilde{a}_{j i} l_{i}\right]\left|z_{i}(t)\right| \\
\leq & 0 .
\end{aligned}
$$

This implies that the zero solution of (7) is globally asymptotically stable, i.e., the equilibrium point $x^{*}=\left(x_{1}^{*}, x_{2}^{*}, \ldots, x_{n}^{*}\right)^{T}$ of $(1)$ is globally asymptotically stable.

When (19) holds,

$$
\left(d_{i}-\tilde{a}_{i i} l_{i}\right)-\sum_{j=1, j \neq i}^{n} \tilde{a}_{i j} l_{j} \geq 0, \quad i=1,2, \ldots, n .
$$


Let

$$
\gamma_{i}=\left(d_{i}-\tilde{a}_{i i} l_{i}\right)-\sum_{j=1, j \neq i}^{n} \tilde{a}_{i j} l_{j} \geq 0, \quad i \in\{1,2, \ldots, n\}
$$

thus

$$
\left(d_{i}-\tilde{a}_{i i} l_{i}\right)-\gamma_{i}=\sum_{j=1, j \neq i}^{n} \tilde{a}_{i j} l_{j}, \quad i \in\{1,2, \ldots, n\},
$$

then there must exist constants $\sigma_{i}>0(i=1,2, \ldots, n)$ such that

$$
\left[\left(d_{j}-\tilde{a}_{j j} l_{j}\right)-\gamma_{j}\right] \sigma_{j}-\sum_{i=1, i \neq j}^{n} \tilde{a}_{i j} l_{j} \sigma_{i}=0, \quad j=1,2, \ldots, n .
$$

The coefficient matrix of (21) is singular, and combining with (20), the existence of $\sigma_{i}>0$ $(i=1,2, \ldots, n)$ can be understood. In fact, some of the references concerning this claim can be found in Liao et al. [21].

From (21),

$$
\left(d_{j}-\tilde{a}_{j j} l_{j}\right) \sigma_{j}-\sum_{i=1, i \neq j}^{n} \tilde{a}_{i j} l_{j} \sigma_{i}=\gamma_{j} \sigma_{j} \geq 0, \quad j=1,2, \ldots, n,
$$

that is,

$$
d_{j}-\sum_{i=1}^{n} \tilde{a}_{i j} l_{j} \sigma_{i} \frac{1}{\sigma_{j}}=\gamma_{j} \geq 0, \quad j=1,2, \ldots, n
$$

Make a full rank linear transform:

$$
w(t)=\operatorname{diag}\left(\sigma_{1}, \sigma_{2}, \ldots, \sigma_{n}\right) z(t) .
$$

From (10), it follows that

$$
\left|\dot{w}_{i}(t)\right| \leq-d_{i}\left|w_{i}(t)\right|+\sum_{j=1}^{n} \sigma_{i} \tilde{a}_{i j} l_{j} \frac{1}{\sigma_{j}}\left|w_{j}\left(t-\tau_{i j}\right)\right|, \quad i \in\{1,2, \ldots, n\} .
$$

Consider the following functional:

$$
\tilde{V}(t)=\sum_{i=1}^{n}\left|w_{i}(t)\right|+\sum_{i=1}^{n} \sum_{j=1}^{n} \int_{t-\tau_{i j}}^{t} \sigma_{i} \tilde{a}_{i j} l_{j} \frac{1}{\sigma_{j}}\left|w_{j}(\vartheta)\right| \mathrm{d} \vartheta .
$$

Calculating the upper right Dini derivative of $\widetilde{V}(t)$ along the trajectory of (24),

$$
\begin{aligned}
D^{+} \widetilde{V}(t) \leq & -\sum_{i=1}^{n} d_{i}\left|w_{i}(t)\right|+\sum_{i=1}^{n} \sum_{j=1}^{n} \sigma_{i} \tilde{a}_{i j} l_{j} \frac{1}{\sigma_{j}}\left|w_{j}\left(t-\tau_{i j}\right)\right| \\
& +\sum_{i=1}^{n} \sum_{j=1}^{n} \sigma_{i} \tilde{a}_{i j} l_{j} \frac{1}{\sigma_{j}}\left|w_{j}(t)\right|-\sum_{i=1}^{n} \sum_{j=1}^{n} \sigma_{i} \tilde{a}_{i j} l_{j} \frac{1}{\sigma_{j}}\left|w_{j}\left(t-\tau_{i j}\right)\right|
\end{aligned}
$$




$$
\begin{aligned}
& =-\sum_{j=1}^{n} d_{j}\left|w_{j}(t)\right|+\sum_{i=1}^{n} \sum_{j=1}^{n} \sigma_{i} \tilde{a}_{i j} l_{j} \frac{1}{\sigma_{j}}\left|w_{j}(t)\right| \\
& =\sum_{j=1}^{n}\left[-d_{j}+\sum_{i=1}^{n} \sigma_{i} \tilde{a}_{i j} l_{j} \frac{1}{\sigma_{j}}\right]\left|w_{j}(t)\right| \\
& \leq 0 .
\end{aligned}
$$

This implies that the zero solution of (7) is globally asymptotically stable, i.e., the equilibrium point $x^{*}=\left(x_{1}^{*}, x_{2}^{*}, \ldots, x_{n}^{*}\right)^{T}$ of $(1)$ is globally asymptotically stable.

Remark 4 The question remains, in fact, what the significance is of the physical parameters of a memristive neurodynamic system to stability. Theorems 1 and 2 make use of the physical parameters of neurodynamic system (1) when proving the stability. These algebraical criteria, which only depend on the parameters of neurodynamic system (1), are easily verified. Theoretically, such stability criteria are very useful because they provide some more efficient ways in analysis and design of a memristive neurodynamic system.

Remark 5 By Theorem 1, we can see that neurodynamic system (1) is globally exponentially stable, if

$$
d_{i}>\sum_{j=1}^{n} \tilde{a}_{i j} l_{j}, \quad i=1,2, \ldots, n, \quad \text { or } \quad d_{i}>\sum_{j=1}^{n} \tilde{a}_{j i} l_{i}, \quad i=1,2, \ldots, n .
$$

From Theorem 2, we know that neurodynamic system (1) is globally asymptotically stable, if

$$
d_{i} \geq \sum_{j=1}^{n} \tilde{a}_{j l} l_{i}, \quad i=1,2, \ldots, n, \quad \text { or } \quad d_{i} \geq \sum_{j=1}^{n} \tilde{a}_{i j} l_{j}, \quad i=1,2, \ldots, n .
$$

Clearly, the critical conditions

$$
d_{i}=\sum_{j=1}^{n} \tilde{a}_{j i} l_{i}, \quad i=1,2, \ldots, n, \quad \text { or } \quad d_{i}=\sum_{j=1}^{n} \tilde{a}_{i j} l_{j}, \quad i=1,2, \ldots, n,
$$

reflect the critical dynamics of memristive neurodynamic system (1).

Remark 6 Recently, some delay-independent stability criteria $[10,12,13]$ and delaydependent stability criteria [14] for memristive neurodynamic systems were reported. Generally, delay-dependent stability criteria are less conservative than delay-independent stability criteria. In order to easily check the stability criteria, stability conditions could best be directly derived from the parameters of the system. Theorems 1 and 2 of this paper, which can be directly derived from the network parameters (without any external parameters), are easily testable. These new results can bring about convenience in applications.

\section{Illustrative examples}

In this section, two numerical examples are given to illustrate the effectiveness of the obtained algebraical criteria. 

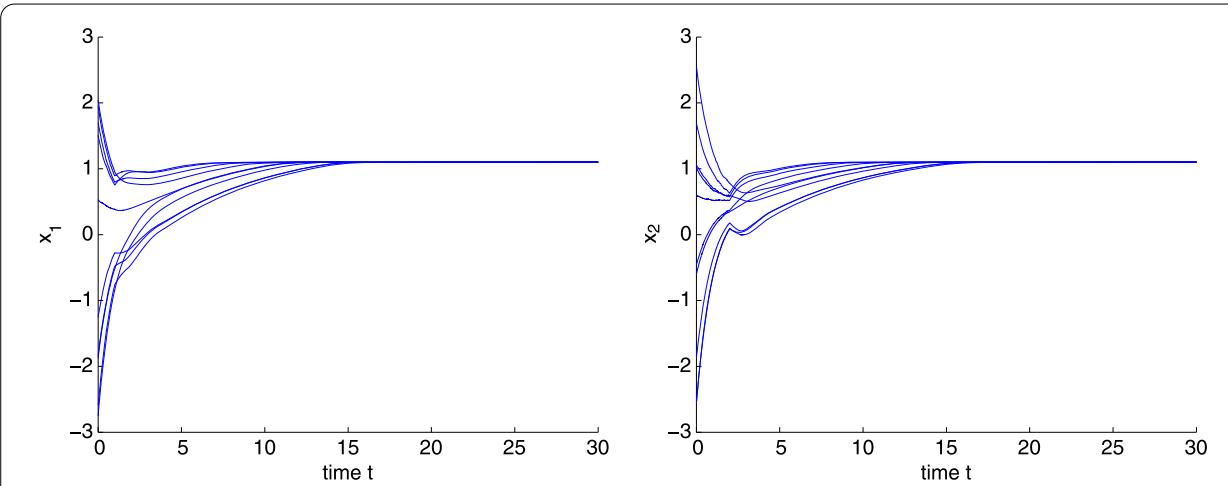

Figure 1 Transient behaviors of trajectories of system (25).

Example 1 Consider a memristive neural network model,

$$
\left\{\begin{array}{l}
\dot{x}_{1}(t)=-x_{1}(t)+a_{11}\left(x_{1}(t)\right) f_{1}\left(x_{1}(t-1)\right)+a_{12}\left(x_{1}(t)\right) f_{2}\left(x_{2}(t-2)\right)+0.3 \\
\dot{x}_{2}(t)=-x_{2}(t)+a_{21}\left(x_{2}(t)\right) f_{1}\left(x_{1}(t-1)\right)+a_{22}\left(x_{2}(t)\right) f_{2}\left(x_{2}(t-2)\right)+0.5
\end{array}\right.
$$

where $f_{i}(v)=\frac{|v+1|-|v-1|}{2}$,

$$
\begin{aligned}
& a_{11}\left(x_{1}(t)\right)=\left\{\begin{array}{ll}
0.4, & x_{1}(t)>0, \\
0.3, & x_{1}(t)<0,
\end{array} \quad a_{12}\left(x_{1}(t)\right)= \begin{cases}0.4, & x_{1}(t)>0, \\
0.3, & x_{1}(t)<0,\end{cases} \right. \\
& a_{21}\left(x_{2}(t)\right)=\left\{\begin{array}{ll}
0.3, & x_{2}(t)>0, \\
0.2, & x_{2}(t)<0,
\end{array} \quad a_{22}\left(x_{2}(t)\right)= \begin{cases}0.3, & x_{2}(t)>0, \\
0.2, & x_{2}(t)<0 .\end{cases} \right.
\end{aligned}
$$

Clearly,

$$
\begin{aligned}
& d_{1}-\sum_{j=1}^{2} \tilde{a}_{1 j}=0.2>0, \\
& d_{2}-\sum_{j=1}^{2} \tilde{a}_{2 j}=0.4>0,
\end{aligned}
$$

the criterion condition (11) in Theorem 1 is satisfied. Figure 1 shows the time-domain behavior of the state variables $x_{1}$ and $x_{2}$ of (25).

Example 2 Consider a memristive neural network model,

$$
\left\{\begin{array}{l}
\dot{x}_{1}(t)=-x_{1}(t)+a_{11}\left(x_{1}(t)\right) f_{1}\left(x_{1}(t-1)\right)+a_{12}\left(x_{1}(t)\right) f_{2}\left(x_{2}(t-2)\right)+3 \\
\dot{x}_{2}(t)=-x_{2}(t)+a_{21}\left(x_{2}(t)\right) f_{1}\left(x_{1}(t-1)\right)+a_{22}\left(x_{2}(t)\right) f_{2}\left(x_{2}(t-2)\right)+5
\end{array}\right.
$$

where $f_{i}(v)=\frac{\exp \{v\}-\exp \{-v\}}{\exp \{v\}+\exp \{-v\}}$,

$$
a_{11}\left(x_{1}(t)\right)=\left\{\begin{array}{ll}
0.7, & x_{1}(t)>0, \\
0.3, & x_{1}(t)<0,
\end{array} \quad a_{12}\left(x_{1}(t)\right)= \begin{cases}0.3, & x_{1}(t)>0 \\
0.1, & x_{1}(t)<0\end{cases}\right.
$$



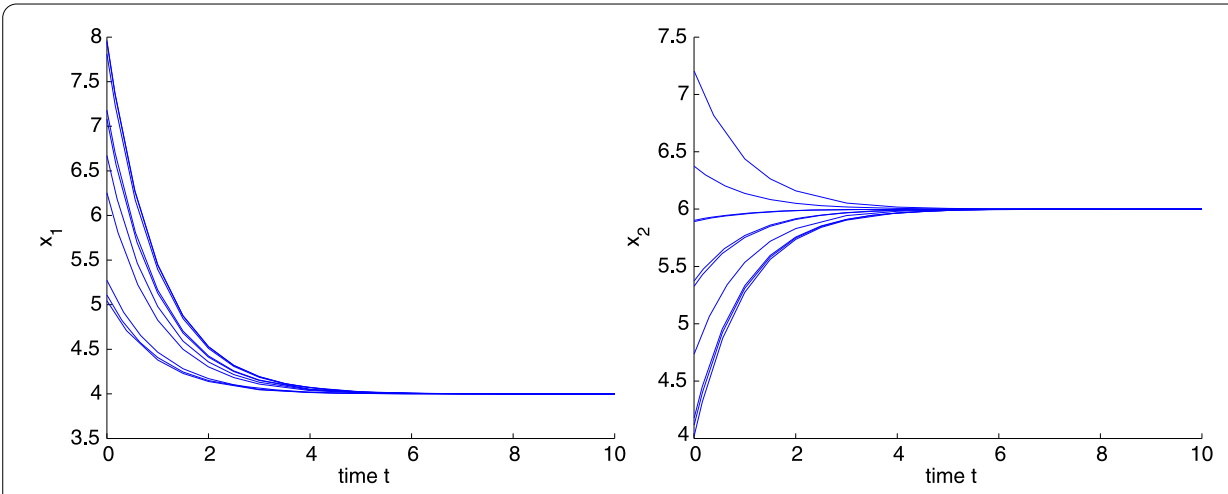

Figure 2 Transient behaviors of trajectories of system (28).

$$
a_{21}\left(x_{2}(t)\right)=\left\{\begin{array}{ll}
0.6, & x_{2}(t)>0, \\
0.4, & x_{2}(t)<0,
\end{array} \quad a_{22}\left(x_{2}(t)\right)= \begin{cases}0.4, & x_{2}(t)>0 \\
0.2, & x_{2}(t)<0 .\end{cases}\right.
$$

Clearly,

$$
\begin{aligned}
& d_{1}-\sum_{j=1}^{2} \tilde{a}_{1 j}=0, \\
& d_{2}-\sum_{j=1}^{2} \tilde{a}_{2 j}=0,
\end{aligned}
$$

the criterion condition (19) in Theorem 2 is satisfied. Figure 2 depicts the time-domain behavior of the state variables $x_{1}$ and $x_{2}$ of (28).

\section{Concluding remarks}

The memristive neurodynamic system exhibits well-characterized analog switching effects in electrical characteristics for devices formed by multiport architectures. Rigorously analyzing the nonlinear dynamics of memristive neurodynamic system is very essential for its neuromorphic computing applications. This paper is concerned with the global exponential stability and global asymptotical stability for a class of delayed memristive neural networks. The newly proposed approach is based on the comparison method and stability theory within a nonsmooth analysis framework. Quite differently, these stability criteria are formulated via some simple algebraical inequalities. The characteristic analysis of memristive neurodynamic systems might also reveal the electrical behavior of analog memristor devices.

\section{Competing interests}

The authors declare that they have no competing interests.

Authors' contributions

All the authors contributed equally to this work. They all read and approved the final version of the manuscript.

\section{Author details}

${ }^{1}$ College of Mathematics and Statistics, Hubei Normal University, Huangshi, 435002, China. ${ }^{2}$ Institute for Information and System Science, Xi'an Jiaotong University, Xi'an, 710049, China. ${ }^{3}$ School of Automation, Huazhong University of Science and Technology, Wuhan, 430074, China. 
Acknowledgements

The work is supported by the Natural Science Foundation of China under Grant 61304057.

\section{Received: 6 January 2015 Accepted: 20 March 2015 Published online: 03 April 2015}

\section{References}

1. Chen, JJ, Zeng, ZG, Jiang, P: Global Mittag-Leffler stability and synchronization of memristor-based fractional-order neural networks. Neural Netw. 51, 1-8 (2014)

2. Chen, JJ, Zeng, ZG, Jiang, P: On the periodic dynamics of memristor-based neural networks with time-varying delays. Inf. Sci. 279, 358-373 (2014)

3. Guo, ZY, Wang, J, Yan, Z: Global exponential dissipativity and stabilization of memristor-based recurrent neural networks with time-varying delays. Neural Netw. 48, 158-172 (2013)

4. Guo, ZY, Wang, J, Yan, Z: Attractivity analysis of memristor-based cellular neural networks with time-varying delays. IEEE Trans. Neural Netw. Learn. Syst. 25(4), 704-717 (2014)

5. Pershin, YV, Di Ventra, M: Experimental demonstration of associative memory with memristive neural networks. Neural Netw. 23(7), 881-886 (2010)

6. Wang, X, Li, CD, Huang, TW: Delay-dependent robust stability and stabilization of uncertain memristive delay neural networks. Neurocomputing 140,155-161 (2014)

7. Wang, X, Li, CD, Huang, TW, Duan, SK: Global exponential stability of a class of memristive neural networks with time-varying delays. Neural Comput. Appl. 24(7-8), 1707-1715 (2014)

8. Wen, SP, Zeng, ZG, Huang, TW: Exponential stability analysis of memristor-based recurrent neural networks with time-varying delays. Neurocomputing 97, 233-240 (2012)

9. Wen, SP, Zeng, ZG, Huang, TW: Dynamic behaviors of memristor-based delayed recurrent networks. Neural Comput. Appl. 23(3-4), 815-821 (2013)

10. $\mathrm{Wu}, \mathrm{AL}$, Zeng, ZG: Dynamic behaviors of memristor-based recurrent neural networks with time-varying delays. Neural Netw. 36, 1-10 (2012)

11. Wu, AL, Zeng, ZG: Exponential stabilization of memristive neural networks with time delays. IEEE Trans. Neural Netw. Learn. Syst. 23(12), 1919-1929 (2012)

12. $\mathrm{Wu}, \mathrm{AL}$, Zeng, ZG: Lagrange stability of memristive neural networks with discrete and distributed delays. IEEE Trans. Neural Netw. Learn. Syst. 25(4), 690-703 (2014)

13. Wu, AL, Zeng, ZG: An improved criterion for stability and attractability of memristive neural networks with time-varying delays. Neurocomputing 145, 316-323 (2014)

14. Wu, AL, Zeng, ZG, Fu, CJ: Dynamic analysis of memristive neural system with unbounded time-varying delays J. Franklin Inst. 351(5), 3032-3041 (2014)

15. Zhang, GD, Shen, Y: New algebraic criteria for synchronization stability of chaotic memristive neural networks with time-varying delays. IEEE Trans. Neural Netw. Learn. Syst. 24(10), 1701-1707 (2013)

16. Zhang, GD, Shen, Y, Yin, Q, Sun, JW: Global exponential periodicity and stability of a class of memristor-based recurrent neural networks with multiple delays. Inf. Sci. 232, 386-396 (2013)

17. Hale, JK, Infante, EF, Tsen, FSP: Stability in linear delay equations. J. Math. Anal. Appl. 105(2), 533-555 (1985)

18. Huang, H, Feng, G, Cao, J: Robust state estimation for uncertain neural networks with time-varying delay. IEEE Trans. Neural Netw. 19(8), 1329-1339 (2008)

19. Huang, TW: Robust stability of delayed fuzzy Cohen-Grossberg neural networks. Comput. Math. Appl. 61(8), 2247-2250 (2011)

20. Huang, TW, Li, CD, Duan, SK, Starzyk, JA: Robust exponential stability of uncertain delayed neural networks with stochastic perturbation and impulse effects. IEEE Trans. Neural Netw. Learn. Syst. 23(6), 866-875 (2012)

21. Liao, XX, Mao, XR, Wang, J, Zeng, ZG: Algebraic conditions of stability for Hopfield neural network. Sci. China, Ser. F, Inf. Sci. 47(1), 113-125 (2004)

22. Liao, XX, Wang, XJ: Stability for differential difference equations. J. Math. Anal. Appl. 173(1), 84-102 (1993)

23. Liu, D, Pang, Z, Lloyd, SR: A neural network method for detection of obstructive sleep apnea and narcolepsy based on pupil size and EEG. IEEE Trans. Neural Netw. 19(2), 308-318 (2008)

24. Shen, Y, Wang, J: Almost sure exponential stability of recurrent neural networks with Markovian switching. IEEE Trans. Neural Netw. 20(5), 840-855 (2009)

25. Shen, $Y$, Wang, J: Robustness analysis of global exponential stability of recurrent neural networks in the presence of time delays and random disturbances. IEEE Trans. Neural Netw. Learn. Syst. 23(1), 87-96 (2012)

26. Wang, D, Liu, D, Wei, Q: Finite-horizon neuro-optimal tracking control for a class of discrete-time nonlinear systems using adaptive dynamic programming approach. Neurocomputing 78(1), 14-22 (2012)

27. Wang, Z, Liu, D: Data-based controllability and observability analysis of linear discrete-time systems. IEEE Trans. Neural Netw. 22(12), 2388-2392 (2011)

28. Wang, Z, Liu, Y, Liu, X: State estimation for jumping recurrent neural networks with discrete and distributed delays. Neural Netw. 22(1), 41-48 (2009)

29. Wu, W, Chen, TP: Global synchronization criteria of linearly coupled neural network systems with time-varying coupling. IEEE Trans. Neural Netw. 19(2), 319-332 (2008)

30. Wu, W, Zhou, WJ, Chen, TP: Cluster synchronization of linearly coupled complex networks under pinning control. IEEE Trans. Circuits Syst. I, Regul. Pap. 56(4), 829-839 (2009)

31. Yi, Z: Foundations of implementing the competitive layer model by Lotka-Volterra recurrent neural networks. IEEE Trans. Neural Netw. 21(3), 494-507 (2010)

32. Yi, Z, Zhang, L, Yu, JL, Tan, KK: Permitted and forbidden sets in discrete-time linear threshold recurrent neural networks. IEEE Trans. Neural Netw. 20(6), 952-963 (2009)

33. Yu, J, Hu, C, Jiang, HJ: $\alpha$-Stability and $\alpha$-synchronization for fractional-order neural networks. Neural Netw. 35, 82-87 (2012)

34. Yu, J, Hu, C, Jiang, HJ, Fan, XL: Projective synchronization for fractional neural networks. Neural Netw. 49, 87-95 (2014)

35. Yu, W, Francisco, PC, Li, X: Two-stage neural sliding mode control of magnetic levitation in minimal invasive surgery. Neural Comput. Appl. 20(8), 1141-1147 (2011) 
36. $\mathrm{Yu}, \mathrm{W}, \mathrm{Li}, \mathrm{X}$ : Automated nonlinear system modeling with multiple fuzzy neural networks and kernel smoothing. Int. J. Neural Syst. 20(5), 429-435 (2010)

37. Zhang, H, Liu, J, Ma, D, Wang, Z: Data-core-based fuzzy min-max neural network for pattern classification. IEEE Trans. Neural Netw. 22(12), 2339-2352 (2011)

38. Zhang, H, Luo, Y, Liu, D: Neural-network-based near-optimal control for a class of discrete-time affine nonlinear systems with control constraints. IEEE Trans. Neural Netw. 20(9), 1490-1503 (2009)

39. Zhang, $\mathrm{H}, \mathrm{Ma}$, T, Huang, $\mathrm{G}$, Wang, Z: Robust global exponential synchronization of uncertain chaotic delayed neural networks via dual-stage impulsive control. IEEE Trans. Syst. Man Cybern., Part B, Cybern. 40(3), 831-844 (2010)

40. Zhang, H, Wang, Y: Stability analysis of Markovian jumping stochastic Cohen-Grossberg neural networks with mixed time delays. IEEE Trans. Neural Netw. 19(2), 366-370 (2008)

41. Cortes, J: Discontinuous dynamical systems. IEEE Control Syst. Mag. 28(3), 36-73 (2008)

Submit your manuscript to a SpringerOpen ${ }^{\odot}$ journal and benefit from:

- Convenient online submission

Rigorous peer review

- Immediate publication on acceptance

- Open access: articles freely available online

- High visibility within the field

- Retaining the copyright to your article 